\title{
On the Spectral Representation of Holomorphic Functions on Some Domain
}

\author{
By \\ Toshihiro WATANABE*
}

\section{§ 0. Introduction}

The investigations of this paper will be concerned with the inverse Fourier-Laplace transform of functions holomorphic in some domain. On this subject the representation of entire functions has been obtained by Paley-Wiener-Schwartz and Eskin (cf.; [2], [7] p. 238). However as for the problem of functions holomorphic in bounded domains, it seems to the author that only the case of a tubular cone has been studied (cf.; [1], [6] Chapter VI Theorem 5, [7] Chapter $\mathrm{V} \S 26)$.

Among these works, Schwartz' theorem characterizes a class of holomorphic functions whose spectral functions ${ }^{\text {t) }} f(x)$ possess the following properties:

$$
\begin{gathered}
\operatorname{supp} f \subset[0, \infty] \\
e^{-(x, \xi)} f(x) \in \mathscr{S}^{\prime}\left(\mathbb{R}^{1}\right) \text { for some } \xi \in \boldsymbol{R}^{1} .
\end{gathered}
$$

Since a distribution in $\mathscr{S}^{\prime}$ is represented in the form of a finite sum of derivatives of continuous functions of power increase, we can say that Schwartz' theorem essentially treats about a spectral function $f(x)$ in $\boldsymbol{R}^{1}$ which satisfies the following properties:

$$
f(x)=\left(\frac{d}{d x}+\xi\right)^{k} f_{0}(x), \operatorname{supp} f_{0} \subset[0, \infty]
$$

for some integer $k \geqq 0$ and constant $\xi \in \boldsymbol{R}^{1}$;

* Communicated by M. Sato, July 7, 1976. Revised February 21, 1977.

Department of Applied Mathematics, University of Gifu, Kagamigahara 504, Japan.

$\dagger$ ) We call the spectral function the inverse Fourier-Laplace transform of functions holomorphic in some domain. 
is a continuous function of power increase.

In this paper, we shall first prove that the Schwartz theorem can be generalized to the case where the spectral function $g(\lambda)$ satisfies the following conditions :

$$
g(\lambda)=\left(\frac{\partial}{\partial \lambda}-y_{0}\right)^{\rho} g_{0}(\lambda), \text { supp } g_{0} \subset \bar{V}^{*} ;
$$

$g_{0}(\lambda)$ is a continuous function and, for any $\varepsilon>0$,

there exists a constant $K_{c}>0$ satisfying the inequality

$$
\left|g_{0}(\lambda)\right| \leqq K_{\varepsilon} \exp \left(\lambda, y_{0}+\varepsilon e\right)
$$

Here $V$ is an affinely homogeneous convex cone in $\boldsymbol{R}^{n}, \bar{V}^{*}$ is the closed dual cone of $V, \rho$ is a multi-index and $\left(\frac{\partial}{\partial \lambda}\right)^{\rho}$ is a RiemannLiouville operator associated with the cone $V$ (see [5], Proposition 1.1, p. 202). Since the support of the fundamental solution of $\left(\frac{\partial}{\partial \lambda}\right)^{\rho}$ is contained in the closed dual cone $\bar{V}^{*}([5]$, Theorem 2.2 p. 216), $\left(\frac{\partial}{\partial \lambda}\right)^{\circ}$ turns out to be a hyperbolic differential operator. Secondly we shall consider the case of the Riemann-Liouville operator $\left(\frac{\partial}{\partial \lambda}-\right.$ $\left.F\left(\frac{\partial}{\partial \zeta}, \frac{\partial}{\partial \zeta}\right)\right)^{\rho}([5]$ Proposition 1. 1, p. 202) associated with the real Siegel domain

$$
D=\left\{(\lambda, \xi) \in \boldsymbol{R}^{n+m}: \lambda-F(\xi, \xi) \in V\right\},
$$

where $F(.,$.$) is a homogeneous V$-positive symmetric bilinear form on $\boldsymbol{R}^{m}$ with values in $\boldsymbol{R}^{n}$. Our result of this case (a main result) characterizes a class of holomorphic functions whose spectral function $f(\lambda, \zeta)$ satisfies the following properties:

$$
f(\lambda, \zeta)=\left(\frac{\partial}{\partial \lambda}-F\left(\frac{\partial}{\partial \zeta}, \frac{\partial}{\partial \zeta}\right)\right)^{\rho} f_{0}(\lambda, \zeta),
$$

where $f_{0}(\lambda, \zeta)$ is continuous in $(\lambda, \zeta) \in \boldsymbol{R}^{n} \times \mathbb{C}^{m}$, entire in $\zeta \in \boldsymbol{C}^{m}$ and is of support in $\bar{V}^{*} \times \boldsymbol{C}^{m}$;

(6) for any $\varepsilon>0$ there exists a constant $K_{\varepsilon}>0$ to satisfy the inequality 


$$
\begin{aligned}
\left|f_{0}(\lambda, \zeta)\right| \leqq & K_{\varepsilon} \lambda_{*}^{-q^{*} / 2} \exp \left\{-\left(\frac{1}{4}-\varepsilon\right) \operatorname{sp} F\left(\hat{\lambda}^{*-1} \xi, \hat{\lambda}^{*-1} \xi\right)\right. \\
& \left.+\left(\frac{1}{4}+\varepsilon\right) \operatorname{sp} F\left(\hat{\lambda}^{*-1} \eta, \hat{\lambda}^{*-1} \eta\right)+\varepsilon \operatorname{sp} \lambda\right\}, \zeta=\xi+i \eta \in \mathbb{C}^{m} .
\end{aligned}
$$

Inequalities (4) and (6) can estimate the fundamental solution of the operators $\left(\frac{\partial}{\partial \lambda}-y_{0}\right)^{\rho}$ and $\left(\frac{\partial}{\partial \lambda}-F\left(\frac{\partial}{\partial \xi}, \frac{\partial}{\partial \xi}\right)\right)^{\circ}$, respectively, where the vectors $\rho=\left(\rho_{1}, \ldots, \rho_{l}\right)$ and $\sigma=\left(\sigma_{1}, \ldots, \sigma_{l}\right)$ satisfy the conditions $\rho_{i}, \sigma_{i}>-d_{i}>0(i=1, \ldots, l)$ for a fixed vector $d=\left(d_{1}, \ldots, d_{l}\right)$. Also the support of $f_{0}(\lambda)$ [resp. $g_{0}(\lambda, \zeta)$ ] is contained in the closed dual cone $\bar{V}^{*}$ [resp. $\bar{V}^{*} \times C^{m}$ ] which is equal to the support of the fundamental solution of the operator $\left(\frac{\partial}{\partial \lambda}-y_{0}\right)^{\rho}\left[\operatorname{resp} \cdot\left(\frac{\partial}{\partial \lambda}-F\left(\frac{\partial}{\partial \xi}, \frac{\partial}{\partial \xi}\right)\right)^{\rho}\right]$. Therefore the spectral function $g(\lambda)$ [resp. $f(\lambda, \zeta)]$ is considered to approximate the fundamental solution of the operator $\left(\frac{\partial}{\partial \lambda}-y_{0}\right)^{\rho}$ $\left[\right.$ resp. $\left.\left(\frac{\partial}{\partial \lambda}-F\left(\frac{\partial}{\partial \xi}, \frac{\partial}{\partial \xi}\right)\right)^{\rho}\right]$. Thus we call holomorphic functions satisfying the conditions (3) and (4) [resp. (5) and (6)] "V-hyperbolic" [resp. "D-parabolic"].

Let us enumerate symbols and notations used in this paper (as for the details of these symbols and notations, see [4], [5]). Let $V$ be an affinely homogeneous convex cone of rank $l$ which does not contain straight lines in $\mathbb{R}^{m}$ and $V^{*}$ be a dual cone of $V$ with respect to the scalar product $(.,$.$) . Since it is possible to transfer to V$ the structure of $T$-algebra ([8], Definition 3, p. 380), we fix a point $e$ in $V$ to satisfy the condition $(e, x)=\operatorname{sp} x([5]$, p. 22, (2.13)) and define the dual vector $x^{*}$ by sp $\left(x^{*} \lambda\right)=(x, \lambda)$. We denote by $\Gamma_{v^{*}}(\rho)$ the gamma function of the cone $V^{*}([5]$, Definition 2.2, p. 22). The symbol $x^{\rho}\left[\right.$ resp. $\left.x_{*}^{\rho}\right]$ is meant by a compound power function of $V$ [resp. $\left.V^{*}\right]$, where $\rho$ is a multi-index ([5], p. 20 (2.3)). Put $\rho^{*}=\left(\rho_{l}, \ldots, \rho_{1}\right)$ for $\rho=\left(\rho_{1}, \ldots, \rho_{l}\right)$. Then we have $x=\left(x^{*}\right)_{*}^{\rho_{*}^{*}}$ ([5], p. 23 (2.26)). The vector $\rho$ for which $x^{\rho}$ becomes a polynomial are called $V$-integral ([5], Definition 3.2, p. 37). $F($,$) denotes a$ homogeneous $V$-positive symmetric bilinear form on $\boldsymbol{R}^{m}$ with values in $\boldsymbol{R}^{n}([4]$, p. 199, (1.1) (1.4)) and also $F($,$) is used in case where$ it is naturally extended on $\boldsymbol{C}^{m}$ with values in $\boldsymbol{C}^{n}$. The vectors $d=\left(d_{i}\right)$ ([5], Proposition 2.2, p. 20), $n=\left(n_{i}\right)$ ([5], p. 14, (1.16)), $q=\left(q_{i}\right)$ 
([4], p. 201, (1.16)), $\hat{\lambda}$ and $\tilde{\lambda}([4]$, p. 212, (2.3), (2.4)) are proper symbols associated with the cone $V$ and the bilinear form $F($,$) .$

\section{§ 1. The Case of "V-hyperbolic" Holomorphic Functions}

In this section we prove the following theorem which generalizes Schwartz' theorem [6] and characterizes the "V-hyperbolicity" of holomorphic functions.

Theorem 1. Let $h(z)$ be a holomorphic function in the tubular cone

$$
T=\left\{z \in C^{n}: \operatorname{lm} z \in V+y_{0}, y_{0} \text { is fixed }\right\} .
$$

Suppose that, for any $\varepsilon>0$, there exist a constant $K_{c}>0$ and a $V$-integral vector $\rho_{0}$ satisfying

$$
|h(z)| \leqq K_{\varepsilon}\left|\left(-i z-y_{0}\right)^{\rho_{0}}\right|
$$

in the closed domain

$$
T_{s}=\left\{z \in C^{n}: \operatorname{lm} z-y_{0}-\varepsilon e \in \bar{V}\right\},
$$

where $\bar{V}$ is the closure of $V$ and $e$ is the identity. Then the spectral function $^{\dagger)} g(\lambda)$ of $h(z)$ is represented for some $V$-integral vector $\rho_{1}$ as

$$
g(\lambda)=\left(\frac{\partial}{\partial \lambda}-y_{0}\right)^{\rho_{1}} g_{0}(\lambda),
$$

where $g_{0}(\lambda)$ is a continuous function with support in the closed dual cone $\bar{V}^{*}$ such that, for any $\varepsilon>0$, there exists a constant $K_{s}^{\prime}>0$ satisfying

$$
\left|g_{0}(\lambda)\right| \leqq K_{\iota}^{\prime} \exp \left(\lambda, y_{0}+e\right) \text {. }
$$

Conversely, if $g(\lambda)$ satisfies these conditions for a V-integral vector $\rho_{1}$ and a fixed vector $y_{0} \in \mathbb{R}^{m}$, then the Fourier-Laplace transform $h(z)$ of $g(\lambda)$ is holomorphic in the tubular domain $T$ and satisfies inequality (1) for a constant $K_{\mathrm{c}}>0$ and a V-integral vector $\rho_{0}$.

Proof. We prepare an equality to use in the proof. Since we

†) As for the definition of the spectral function of the holomorphic function in the tubular cone, see [7], p. 230. 
have

$$
\begin{aligned}
& \int_{V} \exp (i(z, \lambda)) \lambda_{*}^{-\rho^{*}+d^{*}} d \lambda \\
& =\int_{V^{*}} \exp \left(-\operatorname{sp}(-i z)^{*} \lambda\right) \lambda_{*}^{-*^{*}+d^{*}} d \lambda \\
& =(-i z)^{\rho} \int_{V^{*}} \exp (-\operatorname{sp} \lambda) \lambda_{*}^{-\rho^{*}+d^{*}} d \lambda \\
& =\Gamma_{V^{*}}\left(-\rho^{*}\right)(-i z)^{\rho}, \operatorname{lm} z \in V, \operatorname{Re} \rho_{i}<-\frac{n_{i}}{2}, \\
& \quad i=1, \ldots, l,
\end{aligned}
$$

the Parseval-Plancherel formula gives

$$
\begin{aligned}
& \int_{R^{n}+i y}\left|\left(-i z-y_{0}\right)^{\rho}\right|^{2} d x \\
& =(2 \pi)^{n}\left|\Gamma_{V^{*}}\left(-\rho^{*}\right)\right|^{-2} \int_{V^{*}} \exp \left\{-2\left(y-y_{0}, \lambda\right)\right\} \lambda^{-2 \operatorname{Re} \rho^{*}+2 d^{*}} d \lambda \\
& =\left.(2 \pi)^{n} \Gamma_{V^{*}}\left(-\rho^{*}\right)\right|^{-2} \Gamma_{V^{*}}\left(-2 \operatorname{Re} p^{*}+d^{*}\right)\left(2 y-2 y_{0}\right)^{2 \operatorname{Re} \rho-d},
\end{aligned}
$$

where $2 \operatorname{Re} \rho_{i}<d_{i}-\frac{n_{i}}{2}(i=1, \ldots, l)$ and $y \in \boldsymbol{R}^{n}$ is chosen so that $z=x+i y \in T$.

Now let $h(z)$ be a holomorphic function in $T$ satisfying (1.1). Then, for a sufficiently large $V$-integral vector, the spectral function $g(\lambda)$ of $h(z)$ is expressed as follows:

$$
\begin{array}{r}
g(\lambda)=\left(\frac{\partial}{\partial \lambda}-y_{0}\right)^{\rho_{1}} \int_{R^{n}+i y} e^{-i\left(\lambda_{2} z\right)} h(z)\left(-i z-y_{0}\right)^{-\rho_{1}} d x \\
(z=x+i y \in T) .
\end{array}
$$

We set

$$
g_{0}(\lambda)=\int_{R^{n}+i y} e^{-i(\lambda, z)} h(z)\left(-i z-y_{0}\right)^{-\rho_{1}} d x .
$$

Then in virtue of the Cauchy theorem the function $g_{0}(\lambda)$ is independent of the plane of integration in the tubular cone $T$ and we obtain from (1.1) and (1.5)

$$
\begin{aligned}
& \left|g_{0}(\lambda)\right| \leqq K_{\mathrm{e}} \exp (\lambda, y) \int_{R^{n}+i y}\left|\left(-i z-y_{0}\right)^{\rho_{0}-\rho_{1}}\right| d x \\
& \quad=K_{\varepsilon} \exp (\lambda, y)(2 \pi)^{n}\left|\Gamma_{V^{*}}\left(-\left(\rho_{0}^{*}-\rho_{1}^{*}\right) / 2\right)\right|^{-2} \\
& \quad \times \Gamma_{\mathrm{V}^{*}}\left(-\operatorname{Re} \rho_{0}^{*}+\operatorname{Re} \rho_{1}^{*}+d^{*}\right)\left(2 y-2 y_{0}\right)^{\operatorname{Re} \rho_{0}-\operatorname{Re} \rho_{1}-d}
\end{aligned}
$$


If $y$ is chosen so that $z=x+i y \in T_{\text {c }}$. Setting $y=y_{0}+\varepsilon e$ in (1.7), we obtain inequality (1.3). If $\lambda \notin \bar{V}^{*}$, there exists $y_{1} \in V+y_{0}$ satisfying $\left(\lambda, y_{1}-y_{0}\right) \lesseqgtr 0$. Therefore if we set $y=t\left(y_{1}-y_{0}\right)+y_{0}$, then $y \in V+y_{0}$ for any $t>0$. Letting $t \rightarrow+\infty$ in (1.7), we see that the right side of (1.7) vanishes. Hence supp $g \subset \bar{V}^{*}$. Since inequality (1.1) and equality (1.5) gives

$$
\begin{aligned}
& \left|e^{-i(\alpha, z)} h(z) /\left(-i z-y_{0}\right)^{\rho_{1}}\right| \\
& \leqq K_{\imath} e^{(2, y)}\left|\left(-i z-y_{0}\right)^{\rho_{0} \rho^{\rho_{1}}}\right| \in L^{1}, \\
& z \in T_{\mathrm{s}},
\end{aligned}
$$

the continuity of $g_{0}(\lambda)$ follows from Lebegue's convergence theorem.

Conversely, suppose that a function $g(\lambda)$ is given for some $V$-integral vector $\rho_{1}$ by (1.2) with $g_{0}(\lambda)$ satisfying inequality (1.3). Let us set

$$
h(z)=\int_{V^{*}} e^{i(\lambda, z)} g(\lambda) d \lambda .
$$

Then we have

$$
\begin{aligned}
h(z) & =\int_{V^{*}} e^{i(\lambda, z)}\left(\frac{\partial}{\partial \lambda}-y_{0}\right)^{\rho_{1}} g_{0}(\lambda) d \lambda \\
& =\left(-i z-y_{0}\right)^{\rho_{1}} \int_{V^{*}} e^{i(\lambda, z)} g_{0}(\lambda) d \lambda .
\end{aligned}
$$

Inequality (1.3) yields

$$
\begin{array}{r}
\left|\int_{V^{*}} e^{i(\lambda, z)} g_{0}(\lambda) d \lambda\right| \leqq K_{a}^{\prime} \int_{V^{*}} e^{-\left(\lambda, y-y_{0}-c e\right)} d \lambda, \\
z=x+i y .
\end{array}
$$

Since the right side of (1.10) is convergent for $y-y_{0}-\varepsilon e \in V$, we have inequality (1.1) in the closed domain $T_{\bullet}$. Let us show that $h(z)$ is a holomorphic function in the tubular cone T. From (1.3), we see that for $y-y_{0}-\varepsilon e \in V$

$$
\begin{array}{r}
\left|e^{i(\lambda, z)} i \lambda_{k} g_{0}(\lambda)\right| \leqq K^{\prime}\left|\lambda_{k}\right| \exp \left(\lambda,-y+y_{0}+\varepsilon e\right) \in L^{1}, \\
\lambda=\left(\lambda_{1}, \ldots, \lambda_{n}\right) .
\end{array}
$$

Then in virtue of Lebesque's convergence theorem we have 


$$
\begin{aligned}
& \frac{\partial}{\partial x^{k}} \int_{V^{*}} e^{i(\lambda, z)} g_{0}(\lambda) d \lambda \\
& =-i \frac{\partial}{\partial y_{k}} \int_{V} e^{i(\lambda, z)} g_{0}(\lambda) d \lambda \\
& =\int_{V} e^{i(\lambda, z)} i \lambda_{k} g_{0}(\lambda) d \lambda,
\end{aligned}
$$

where $z=\left(z_{1}, \ldots, z_{n}\right) \in C^{n}, z_{k}=x_{k}+i y_{k}$ and $\lambda=\left(\lambda_{1}, \ldots, \lambda_{n}\right)$ and also each integration of (1.12) is continuous in $x_{k}$ and $y_{k}(k=1, \ldots, n)$. Since the first equality of (1.12) is the Cauchy-Riemann equation, we conclude that $h(z)$ is holomorphic in the domain $\mathrm{T}$.

Q. E. D.

\section{§ 2. The Case of "D-parabolic" Holomorphic Functions}

In order to characterize the "D-parabolicity" of holomorphic functions, we prepare a lemma.

Lemma. Let $h(u)$ be an entire function in $\boldsymbol{C}^{m}$ which, for any $\varepsilon>0$, satisfies the following:

$$
\begin{aligned}
|h(u)| & \leqq K_{\varepsilon} \exp \left\{-(1-\varepsilon)\left(\lambda, F\left(u_{1}, u_{1}\right)\right)^{p_{1} / 2}\right. \\
& \left.+(1+\varepsilon)\left(\lambda, F\left(u_{2}, u_{2}\right)\right)^{p_{2} / 2}\right\}
\end{aligned}
$$

where $\lambda \in V^{*}, p_{i} \supsetneqq 1(i=1,2)$ and $u=u_{1}+i u_{2} \in C^{m}$. Then the spectral function $f(\zeta)$ of $h(u)$ :

$$
f(\zeta)=\int_{R^{m+i u_{2}}} e^{-i \mathrm{SpF}(\zeta, u)} h(u) d u_{1}
$$

is entire and for any $\varepsilon>0$ satisfies the inequality

$$
\begin{aligned}
|f(\zeta)| & \leqq K_{\varepsilon}^{\prime} \lambda_{*}^{-q^{*} / 2} \exp \left\{-\left(p_{2}^{\prime-1} p_{2}^{-p_{2}^{\prime} / p_{2}}-\varepsilon\right)\right. \\
& \times\left(\operatorname{sp} F\left(\hat{\lambda}^{*-1} \xi, \hat{\lambda}^{*-1} \xi\right)\right)^{p_{2}^{\prime} / 2} \\
& \left.+\left(p_{1}^{\prime-1} p_{1}^{-p_{1}^{\prime} / p_{1}}+\varepsilon\right)\left(\operatorname{sp} F\left(\hat{\lambda}^{*-1} \eta, \hat{\lambda}^{*-1} \eta\right)\right)^{p_{1}^{\prime} / 2}\right\},
\end{aligned}
$$

where $1 / p_{i}+1 / p_{i}^{\prime}=1 \quad(i=1,2)$ and $\zeta=\xi+i \eta \in C^{m}$.

Conversely, if $f(\zeta)$ satisfies these conditions for certain numbers $p_{i}>1(i=1,2)$, then its Fourier-Laplace transform $h(u)$ : 


$$
h(u)=\int_{R^{m}+i \eta} e^{i \mathrm{sp} F(u, \xi)} f(\zeta) d \xi
$$

is entire and satisfies inequality (2.1) for any $\varepsilon>0$.

Proof. From inequality (2.1) we have

$$
\begin{aligned}
& |f(\zeta)| \leqq K_{\varepsilon} \exp \left\{\operatorname{sp} F\left(\xi, u_{2}\right)+(1+\varepsilon)\left(\lambda, F\left(u_{2}, u_{2}\right)\right)^{p_{2} / 2}\right\} \\
& \times \int_{R^{m}} \exp \left\{\operatorname{sp} F\left(\eta, u_{1}\right)-(1-\varepsilon)\left(\lambda, F\left(u_{1}, u_{1}\right)\right)^{p_{1} / 2}\right\} d u_{1} .
\end{aligned}
$$

Put $u_{1}^{\prime}=\tilde{\lambda}^{*} u_{1}$. Then the integral of the right side of (2.3) becomes

$$
\begin{aligned}
& \int_{R^{m}} \exp \left\{\operatorname{sp} F\left(\eta, u_{1}\right)-(1-\varepsilon)\left(\lambda, F\left(u_{1}, u_{1}\right)\right)^{p_{1} / 2}\right\} d u_{1} \\
& =\int_{R^{m}} \exp \left\{\operatorname{sp} F\left(\eta, u_{1}\right)-(1-\varepsilon)\left(\operatorname{sp} F\left(\tilde{\lambda}^{*} u_{1}, \tilde{\lambda}^{*} u_{1}\right)\right)^{p_{1} / 2}\right\} d u_{1} \\
& =\lambda_{*}^{-q^{*} / 2} \int_{R^{m}} \exp \left\{\operatorname{sp} F\left(\tilde{\lambda}^{*-1} \eta, u_{1}^{\prime}\right)-(1-\varepsilon)\left(\operatorname{sp} F\left(u_{1}^{\prime}, u_{1}^{\prime}\right)\right)^{p_{1} / 2}\right\} d u_{1}^{\prime} .
\end{aligned}
$$

Putting

$$
r=\left(\operatorname{sp} F\left(u_{1}^{\prime}, u_{1}^{\prime}\right)\right)^{1 / 2} \text { and } s=\left(\operatorname{sp} F\left(\hat{\lambda}^{*-1} \eta, \hat{\lambda}^{*-1} \eta\right)\right)^{1 / 2}
$$

and using the Schwarz inequality, we have

$$
\begin{aligned}
& \lambda_{*}^{-q^{*} / 2} \int_{R_{m}} \exp \left\{\operatorname{sp} F\left(\hat{\lambda}^{*-1} \eta, u_{1}^{\prime}\right)-(1-\varepsilon)\left(\operatorname{sp} F\left(u_{1}^{\prime}, u_{1}^{\prime}\right)\right)^{p_{1} / 2}\right\} d u_{1}^{\prime} \\
& \leqq C_{6} \lambda_{*}^{-q^{*} / 2} \sup _{0 \leqq r<\infty}\left\{\exp \left(r \cdot s-(1-\varepsilon) r^{p_{1}}\right)\right\} .
\end{aligned}
$$

In virtue of the Young inequality we can estimate the right side of (2.5) as follows :

$$
\begin{aligned}
& C_{\varepsilon} \lambda_{*}^{-q^{*} / 2} \sup _{0 \leqq r<\infty}\left\{\exp \left(r \cdot s-(1-\varepsilon) r^{p_{1}}\right)\right\} \\
& \leqq C_{8}^{\prime} \lambda_{*}^{-q^{*} / 2} \exp \left\{\left({p_{1}^{\prime}-1}^{-1} p_{1}^{-p_{1}^{\prime} / p_{1}}+\varepsilon\right) s^{p_{1}^{\prime}}\right\}
\end{aligned}
$$

Summing up, we obtain

$$
\begin{aligned}
& \int_{R^{m}} \exp \left\{\operatorname{sp} F\left(\eta, u_{1}\right)-(1-\varepsilon)\left(\lambda, F\left(u_{1}, u_{1}\right)\right)^{p_{1} / 2}\right\} d u_{1}
\end{aligned}
$$

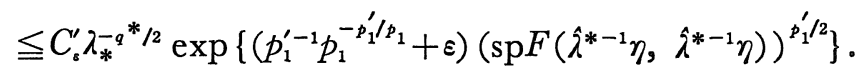

Since $h(u)$ is an entire function, the spectral function $f(\zeta)$ is inde- 
pendent of $u_{2}=\operatorname{Im} u$. So we put

$$
u_{2}=-p_{2}^{-p_{2}^{\prime} / p_{2}}\left(\operatorname{sp} F\left(\hat{\lambda}^{*-1} \xi, \hat{\lambda}^{*-1} \xi\right)\right)^{1 / 2\left(p_{2}^{\prime} / p_{2}-1\right)} \tilde{\lambda}^{*-1} \hat{\lambda}^{*-1} \xi
$$

in inequality (2.3). Then by setting $t=\left(\operatorname{sp} F\left(\hat{\lambda}^{*-1} \xi, \hat{\lambda}^{*-1} \xi\right)\right)^{1 / 2}$, we have

$$
\begin{aligned}
& \exp \left\{\operatorname{sp} F\left(\xi, u_{2}\right)+(1+\varepsilon)\left(\lambda, F\left(u_{2}, u_{2}\right)\right)^{p_{2} / 2}\right\} \\
& =\exp \left\{-p_{2}^{-p_{2}^{\prime} / p_{2}} t^{\left(p_{2}^{\prime} / p_{2}+1\right)}+(1+\varepsilon) p_{2}^{-p_{2}^{\prime}} t^{p_{2}^{\prime}}\right\} \\
& =\exp \left\{\left((1+\varepsilon) p_{2}^{-p_{2}^{\prime}}-p_{2}^{-p_{2}^{\prime} / p_{2}}\right) t^{p_{2}^{\prime}}\right\} \\
& =\exp \left\{p_{2}^{-p_{2}^{\prime} / p_{2}}\left(p_{2}^{-p_{2}^{\prime}+p_{2}^{\prime} / p_{2}}-1+\varepsilon\right) t^{p^{\prime}}\right\} \\
& =\exp \left\{p_{2}^{-p_{2}^{\prime} / p_{2}}\left(p_{2}^{-1}-1+\varepsilon\right) t^{p_{2}^{\prime}}\right\} \\
& =\exp \left\{-\left(p_{2}^{\prime-1} p_{2}^{-p_{2}^{\prime} / p_{2}}-\varepsilon\right)\left(\operatorname{sp} F\left(\hat{\lambda}^{*-1} \xi, \hat{\lambda}^{*-1} \xi\right)\right)^{p_{2}^{\prime} / 2}\right\} .
\end{aligned}
$$

Inequalities (2.3) and (2.7) and equality (2.8) prove (2.2). The analyticity of $f(\zeta)$ can be proved by a way similar to the proof of Theorem 1.

Conversely, suppose that $f(\zeta)$ is an entire function satisfying inequality (2.2) for any $\varepsilon>0$. Then the Fourier-Laplace transform $h(u)$ of $f(\zeta)$ :

$$
h(u)=\int_{R^{m}+i \eta} e^{i \mathrm{spF}(u, \zeta)} f(\zeta) d \xi \quad \zeta=\xi+i \eta,
$$

can be estimated as follows:

$$
\begin{aligned}
|h(u)| & \leqq K_{\mathrm{s}} \exp \left\{-\operatorname{sp} F\left(u_{1}, \eta\right)\right. \\
& \left.+\left(p_{1}^{\prime-1} p_{1}^{-p_{1}^{\prime} p_{1}}+\varepsilon\right)\left(\operatorname{sp} F\left(\hat{\lambda}^{*-1} \eta, \hat{\lambda}^{*-1} \eta\right)\right)^{p_{1}^{\prime} / 2}\right\} \\
& \times \int_{R^{m}} \lambda_{*}^{-q^{*} / 2} \exp \left\{-\operatorname{sp} F\left(u_{2}, \xi\right)-\left({p_{2}^{\prime}}^{-1} p_{2}^{-p_{2}^{\prime} / p_{2}}-\varepsilon\right)\right. \\
& \left.\times\left(\operatorname{sp} F\left(\hat{\lambda}^{*-1} \xi, \hat{\lambda}^{*-1} \xi\right)\right)^{p_{2}^{\prime} / 2}\right\} d \xi .
\end{aligned}
$$

Putting $\xi^{\prime}=\hat{\lambda}^{*-1} \xi$ in the integral of (2.9), we have

$$
\begin{aligned}
& \int_{R^{m}} \lambda_{*}^{-q^{*} / 2} \exp \left\{-\operatorname{sp} F\left(u_{2}, \xi\right)\right. \\
&\left.\quad-\left(p_{2}^{\prime-1} p_{2}^{-p_{2}^{\prime} / \rho_{2}}-\varepsilon\right)\left(\operatorname{sp} F\left(\hat{\lambda}^{*-1} \xi, \hat{\lambda}^{*-1} \xi\right)\right)^{p_{2}^{\prime} / 2}\right\} d \xi \\
&=\int_{R^{m}} \exp \left\{-\operatorname{sp} F\left(\tilde{\lambda}^{*} u_{2}, \xi^{\prime}\right)\right. \\
&\left.\quad-\left(p_{2}^{\prime-1} p_{2}^{-p_{2}^{\prime} / p_{2}}-\varepsilon\right)\left(\operatorname{sp} F\left(\xi^{\prime}, \xi^{\prime}\right)\right)^{p_{2}^{\prime} / 2}\right\} d \xi^{\prime} .
\end{aligned}
$$


Further putting

$$
a=\left(\operatorname{sp} F\left(\tilde{\lambda}^{*} u_{2}, \tilde{\lambda}^{*} u_{2}\right)\right)^{1 / 2} \text { and } b=\left(\operatorname{sp} F\left(\xi^{\prime}, \xi^{\prime}\right)\right)^{1 / 2},
$$

and using the Schwarz inequality, we obtain

$$
\begin{aligned}
& \int_{R^{m}} \exp \left\{-\operatorname{sp} F\left(\tilde{\lambda}^{*} u_{2}, \xi^{\prime}\right)\right. \\
&\left.\quad\left(p_{2}^{\prime-1} p_{2}^{-p_{2}^{\prime} / p_{2}}-\varepsilon\right)\left(\operatorname{sp} F\left(\xi^{\prime}, \xi^{\prime}\right)\right)^{p_{2}^{\prime} / 2}\right\} d \xi^{\prime} \\
& \leqq C_{\varepsilon} \sup _{0 \leqq b<\infty}\left\{\exp \left(a \cdot b-\left(p_{2}^{\prime-1} p_{2}^{-p_{2}^{\prime} / p_{2}}-\varepsilon\right) b^{p_{2}^{\prime}}\right)\right\} .
\end{aligned}
$$

The Young inequality yields the inequality

$$
\begin{aligned}
& C_{\varepsilon} \sup _{0 \leq b<\infty}\left\{\exp \left(a \cdot b-\left(p_{2}^{\prime-1} p_{2}^{-p_{2}^{\prime} / p_{2}}-\varepsilon\right) b^{p_{2}^{\prime}}\right)\right\} \\
& \leqq C_{c}^{\prime} \exp \left\{(1+\varepsilon) a^{p_{2}}\right\}
\end{aligned}
$$

Consequently, we obtain

$$
\begin{aligned}
& \int_{R^{m}} \lambda_{*}^{-q^{*} / 2} \exp \left\{-\operatorname{sp} F\left(u_{2}, \xi\right)\right. \\
& \left.\quad-\left(p_{2}^{\prime-1} p_{2}^{-p_{2}^{\prime} / p_{2}}-\varepsilon\right)\left(\operatorname{sp} F\left(\lambda^{*-1} \xi, \lambda^{*-1} \xi\right)\right)^{p_{2}^{\prime} / 2}\right\} d \xi \\
& \leqq C_{\varepsilon}^{\prime} \exp \left\{(1+\varepsilon)\left(\lambda, F\left(u_{2}, u_{2}\right)\right)^{p_{2} / 2}\right\} .
\end{aligned}
$$

On the other hand the function $h(u)$ is independent of the plane of integration $\zeta=\xi+i \eta$ (where $\eta=$ constant). Therefore by setting

$$
\eta=p_{1}\left(\lambda, F\left(u_{1}, u_{1}\right)\right)^{1 / 2\left(p_{1} / p_{1}^{\prime}-1\right)} \hat{\lambda}^{*} \tilde{\lambda}^{*} u_{1} \text { and } c=\left(\lambda, F\left(u_{1}, u_{1}\right)\right)^{1 / 2},
$$

we have

$$
\begin{aligned}
\exp \{ & -\operatorname{sp} F\left(u_{1}, \eta\right)+\left(p_{1}^{\prime-1} p_{1}^{-p_{1}^{\prime} / p_{1}}+\varepsilon\right) \\
& \left.\times\left(\operatorname{sp} F\left(\hat{\lambda}^{*-1} \eta, \hat{\lambda}^{*-1} \eta\right)\right)^{p_{1}^{\prime}}\right\} \\
= & \exp \left\{-p_{1} c^{p_{1} / p_{1}^{\prime}+1}+\left({p_{1}^{\prime}-1}^{-p_{1}^{p_{1}^{\prime}} p_{1}+p_{1}^{\prime}}+\varepsilon\right) c^{p_{1}}\right\} \\
= & \exp \left\{\left(p_{1}^{\prime-1} p_{1}-p_{1}+\varepsilon\right) c^{p_{1}}\right\} \\
= & \exp \left\{-(1-\varepsilon)\left(\lambda, F\left(u_{1}, u_{1}\right)\right)^{p_{1} / 2}\right\} .
\end{aligned}
$$

Thus inequality (2.1) follows from (2.9), (2.13) and (2.14). The analyticity of $f(\zeta)$ can be proved by a way similar to the proof of Theorem 1. Q. E. D. 
Now we can state the main result concerning the "D-parabolicity" of holomorphic functions.

Theorem 2. Let $h(z, u)$ be a holomorphic function in the domain

$$
\begin{aligned}
D= & \left\{(z, u) \in C^{n+m}: \operatorname{lm} z+F\left(u_{1}, u_{1}\right)-F\left(u_{2}, u_{2}\right) \in V,\right. \\
& \left.u=u_{1}+i u_{2} \in C^{m}\right\} .
\end{aligned}
$$

Suppose, for any $\varepsilon>0$, there exist a constant $C_{\mathbf{c}}>0, a$-integral vector $\rho_{0}$ and integers $k_{i}>0(i=1,2)$ such that

$$
\begin{aligned}
& |h(z, u)| \leqq C_{\varepsilon}\left\{\left(1+\operatorname{sp} F\left(u_{1}, u_{1}\right)+\operatorname{sp} F\left(u_{2}, u_{2}\right)\right)^{k_{1}}\right. \\
& \left.\times\left|(-i z+F(u, u))^{\rho_{0}}\right|+\left(1+\operatorname{sp} F\left(u_{1}, u_{1}\right)+\operatorname{sp} F\left(u_{2}, u_{2}\right)\right)^{k_{2}}\right\}
\end{aligned}
$$

in the closed domain

$$
D_{\varepsilon}=\left\{(z, u) \in C^{n+m}: \operatorname{lm} z+F\left(u_{1}, u_{1}\right)-F\left(u_{2}, u_{2}\right)-\varepsilon e \in \bar{V}\right\} .
$$

Then, for some V-integral vector $\rho_{1}$, the spectral function

$$
\begin{gathered}
f(\lambda, \zeta)=\int_{R^{m}+i u_{2}} \int_{R^{m}+i y} e^{-i(\lambda, z)-i \operatorname{spF}(\zeta, u)} h(z, u) d x d u_{1} \\
z=x+i y \in C^{n}, u=u_{1}+i u_{2} \in C^{m}
\end{gathered}
$$

is represented as

$$
f(\lambda, \zeta)=\left(\frac{\partial}{\partial \lambda}-F\left(\frac{\partial}{\partial \zeta}, \frac{\partial}{\partial \zeta}\right)\right)^{\rho_{1}} f_{0}(\lambda, \zeta),
$$

where the function $f_{0}(\lambda, \zeta)$ is continuous in $(\lambda, \zeta) \in \boldsymbol{R}^{n} \times \boldsymbol{C}^{m}$, entire in $\zeta \in C^{m}$ and is of support in $\bar{V}^{*} \times C^{m}$, and satisfies in $V^{*} \times C^{m}$ the inequality

$$
\begin{gathered}
\left|f_{0}(\lambda, \zeta)\right| \leqq K_{\mathrm{s}} \lambda_{*}^{-q^{*} / 2} \exp \left\{-\left(\frac{1}{4}-\varepsilon\right) \operatorname{sp} F\left(\hat{\lambda}^{*-1} \xi, \lambda^{*-1} \xi\right)\right. \\
\left.+\left(\frac{1}{4}+\varepsilon\right) \operatorname{sp} F\left(\hat{\lambda}^{*-1} \eta, \hat{\lambda}^{*-1} \eta\right)+\varepsilon \operatorname{sp} \lambda\right\}, \\
\zeta=\xi+i \eta \in C^{m} .
\end{gathered}
$$

Conversely, if a function $f(\lambda, \zeta)$ satisfies these conditions (2.16) and (2.17) for any $\varepsilon>0$ and some V-integral $\rho_{1}$, the Fourier-Laplace transform

$$
h(z, u)=\int_{R^{m}+i \eta} \int_{V} e^{i(\lambda, z)+i \operatorname{sp} F(u, \zeta)} f(\lambda, \zeta) d \lambda d \xi
$$


is holomorphic in the domain $D$ and satisfies the inequality

$$
|h(z, u)| \leqq C_{\circ}^{\prime}\left|(-i z+F(u, u))^{\rho_{1}}\right|
$$

in the closed domain

$$
\begin{aligned}
D_{\varepsilon}^{\prime} & =\left\{(z, u) \in C^{n+m}: \operatorname{lm} z+(1-\varepsilon) F\left(u_{1}, u_{1}\right)\right. \\
& \left.-(1+\varepsilon) F\left(u_{2}, u_{2}\right)-\varepsilon e \in \bar{V}\right\} \subset D \text { for any } \varepsilon>0 .
\end{aligned}
$$

Proof. We denote by $g(\lambda, u)$ the spectral function of $h(z, u)$ with respect to $z$ :

$$
g(\lambda, u)=\int_{R^{n}+i y} e^{-i(\lambda, z)} h(z, u) d x \quad(z=x+i y)
$$

where, for any fixed $u \in C^{m}, y$ is chosen so that $(z, u) \in D$. Then for any $V$-integral vector $\rho_{1}$,

$$
\begin{aligned}
g(\lambda, u) & =\left(\frac{\partial}{\partial \lambda}+F(u, u)\right)^{\rho_{1}} \\
& \times \int_{R^{n}+i y} e^{-i(\lambda, z)} h(z, u)(-i z+F(u, u))^{-\rho_{1}} d x \quad(z, u) \in D .
\end{aligned}
$$

Put

$$
g_{0}(\lambda, u)=\int_{R^{n}+i y} e^{-i(\lambda, z)} h(z, u)(-i z+F(u, u))^{-\rho_{1}} d x
$$

Then by Theorem 1 we see that $g_{0}(\lambda, u)$ is continuous in $\lambda \in \boldsymbol{R}^{n}$ and the support of $g_{0}(\lambda, u)$ is contained in $\bar{V}^{*} \times \boldsymbol{C}^{m}$. Since, for any fixed $u \in \boldsymbol{C}^{m}$, the plane of integration of (2.20) is chosen so that $(z, u) \in D, g_{0}(\lambda, u)$ is an entire function of $u \in C^{m}$. Since the integrand $h(z, u)(-i z+F(u, u))^{-\rho_{1}}$ of $(2.20)$ is holomorphic in the domain $D$, we see that $g_{0}(\lambda, u)$ is independent of $y_{\text {. Therefore it }}$ follows from (1.5), (2.15) and (2.20) that for a sufficiently large $V$-integral vector $\rho_{1}$ and $y=-F\left(u_{1}, u_{1}\right)+F\left(u_{2}, u_{2}\right)+\varepsilon e(\varepsilon>0)$,

$$
\begin{aligned}
\left|g_{0}(\lambda, u)\right| \leqq & C_{c}^{\prime}\left(1+\operatorname{sp} F\left(u_{1}, u_{1}\right)+\operatorname{sp} F\left(u_{2}, u_{2}\right)\right)^{k_{3}} \\
& \times \exp \left(\lambda,-F\left(u_{1}, u_{1}\right)+F\left(u_{2}, u_{2}\right)+\varepsilon e\right) \\
\leqq & C_{c}^{\prime \prime} \exp \left\{-(1-\varepsilon)\left(\lambda, F\left(u_{1}, u_{1}\right)\right)\right. \\
& \left.+(1+\varepsilon)\left(\lambda, F\left(u_{2}, u_{2}\right)\right)+\varepsilon \operatorname{sp} \lambda\right\} .
\end{aligned}
$$

Now if we apply Lemma with $p_{1}=p_{2}=2$ to the function 


$$
f_{0}(\lambda, \zeta)=\int_{R^{m}+i u_{2}} e^{-i \operatorname{sp} F(\zeta, u)} g_{0}(\lambda, u) d u_{1}
$$

then we see from (2. 2) that $f_{0}(\lambda, \zeta)$ satisfies inequality (2.17) and is an entire function of $\zeta \in C^{n}$. The continuity of the function $f_{0}(\lambda, \zeta)$ with respect to $\lambda \in \boldsymbol{R}^{n}$ is obvious. We have from (2.19)

$$
\begin{aligned}
f(\lambda, \zeta) & =\int_{R^{n}+i u_{2}} e^{-i \operatorname{sp} F(\zeta, u)} g(\lambda, u) d u_{1} \\
& =\left(\frac{\partial}{\partial \lambda}-F\left(\frac{\partial}{\partial \zeta}, \frac{\partial}{\partial \zeta}\right)\right)^{\rho_{1}} f_{0}(\lambda, \zeta) .
\end{aligned}
$$

Conversely, if the function $f(\lambda, \zeta)$ satisfies conditions (2.16) and (2.17) for any $\varepsilon>0$ and some $V$-integral vector $\rho_{1}$, it follows from Lemma with $p_{1}=p_{2}=2$ that $g_{0}(\lambda, u)$ in (2.22) is continuous in $\lambda \in \mathbb{R}^{n}$ and entire in $\zeta \in C^{m}$, and satisfies the inequality

$$
\begin{aligned}
\left|g_{0}(\lambda, u)\right| \leqq K_{\varepsilon}^{\prime} \exp \left\{-(1-\varepsilon)\left(\lambda, F\left(u_{1}, u_{1}\right)\right)\right. \\
\left.+(1+\varepsilon)\left(\lambda, F\left(u_{2}, u_{2}\right)\right)+\varepsilon \operatorname{sp} \lambda\right\} .
\end{aligned}
$$

Then, by use of Theorem 1, the Fourier-Laplace transform $h(z, u)$ of $f(\lambda, \zeta)$ is holomorphic in the domain $\mathrm{D}$ and satisfies (2.18) in the domain $D_{s}^{\prime}$.

Q. E. D.

\section{References}

[1] Bochner, S., Group invariance of Cauchy's formula in several variables, Ann. of Math. 45 (1944), 686-707.

[2] Eskin, G.I., Obobshcheniye teoremy Paleya-Vinera- Shvartsa, Uspehi Math. Nauk, XVI (1961), no. 1(97), 185-188.

[3] Eidelman, S. D., Parabolic Systems, North Holland, 1969.

[4] Gindikin, S. G., The Cauchy problem for strongly homogeneous differential operators, Trudy Moskov. Mat. Obšč. 16 (1967), 181-208 (Trans. of Moscow Math. Soc. 16 (1967), 197-225.)

[5] Gindikin, S. G., Analysis in homogeneous domains, Uspehi Mat. Nauk, 19 (1964), no. 4 (118), 3-92 (Russian Math. Surveys, 19 (1964), 1-89.)

[6] Schwartz, L., Méthodes Mathématiques pour les Sciences Physiques, Hermann, Paris, 1961.

[7] Vladimirov, V.S., Methods of the Theory of Functions of Sevcral Complex Variables, M. I. T. Press, Cambridge, Mass., England, (1966).

[8] Vinberg, E. B., The theory of homogeneous convex cones, Trudy Moskov. Mat. Obšc. 12 (1963), 303-358 (Trans. Moscow Math. Soc. (1963), 340-403.)

[9] Watanabe, T., On distributions measured by the Riemann-Liouville operators associated with homogeneous convex cones, Hiroshima Math.J. 7 (1977), 643-653. 
